\title{
Autonomic dysfunction: A comparative study of patients with Alzheimer's and frontotemporal dementia - A pilot study
}

Thomas Gregor Issac, Sadanandavalli Retnaswami Chandra ${ }^{1}$, Neelesh Gupta ${ }^{1}$, Malligurki Raghurama Rukmani ${ }^{2}$, S. Deepika, T. N. Sathyaprabha ${ }^{2}$

Departments of Clinical Neurosciences, ${ }^{1}$ Neurology and ${ }^{2}$ Neurophysiology, National Institute of Mental Health and Neurosciences, Bengaluru, Karnataka, India

\begin{abstract}
Introduction: In frontotemporal dementia (FTD) and Alzheimer's disease (AD), central autonomic structures get affected early. An insight into autonomic functions in these patients is likely to be of diagnostic importance and thus help in prognosticating and also probably explain unexplained sudden death in some of these patients. Objectives: The objective of this study is to identify autonomic dysfunction prevailing in patients. Then, if there is dysfunction, is the pattern same or different in these two conditions. And if different it will serve as an additional biomarker for specific diagnosis. Patients and Methods: There were 25 patients and 25 controls and six patients and three controls in AD and FTD groups, respectively. The participants who were recruited were assessed for heart rate variability and conventional cardiac autonomic function testing. The parameters were analyzed using LabChart version 7 software and compared with control population using appropriate statistical methods using SPSS version 22 software. Results: The mean overall total power was low in the FTD group $(P<0.001)$, and there was significant reduction in the standard deviation of normal-to-normal intervals and root mean square of successive differences $(P<0.001)$ with elevated sympathovagal balance in the FTD group $(P=0.04)$. Patients with $\mathrm{AD}$ also showed sympathetic dominance, but there was in addition parasympathetic suppression unlike in the FTD group. Conclusion: This study reveals autonomic dysfunction in patients with FTD and AD. Both conditions show sympathetic dominance, probably consecutive to the involvement of central autonomic regulatory structures as a shared domain. It remains to be confirmed if these findings are the cause or effect of neurodegeneration and might open up newer territories of research based on the causal role of neurotransmitters in these regions and thus lead to novel therapeutic options such as yoga. The presence of parasympathetic suppression in $\mathrm{AD}$ in addition helps differentiate these two conditions.
\end{abstract}

Key words: Alzheimer's disease, autonomic function tests, frontotemporal dementia, heart rate variability, sympathovagal balance

\section{Introduction}

Alzheimer's disease (AD) and frontotemporal dementia (FTD) are neurodegenerative diseases, with a prevalence of $60-80 \%$ of all dementias in the former followed by $10-20 \%$ in the later ${ }^{[1,2]}$ Early diagnosis

\section{Address for correspondence:}

Dr. Sadanandavalli Retnaswami Chandra, Faculty Block,

Neurocentre, National Institute of Mental Health and

Neurosciences, Bengaluru - 560 029, Karnataka, India.

E-mail: drchandrasasi@yahoo.com

\begin{tabular}{|l|l|}
\hline \multicolumn{2}{|c|}{ Access this article online } \\
\hline Quick Response Code: & Website: \\
\hline & www.ruralneuropractice.com \\
\cline { 2 - 3 } & \\
\hline
\end{tabular}

is important to improve the quality of life. However, all available tools are inefficient in the early stages to pinpoint the type to which it will evolve. There are early personality changes in FTD and amnesia as well as visuospatial disorientation in $\mathrm{AD}$ in addition to a spectrum of cognitive dysfunctions. ${ }^{[1,2]}$ The incidence is on the rise and causes burden to patient and family.

Central autonomic structures including hypothalamus, amygdala insula, and locus coeruleus are some of the

This is an open access article distributed under the terms of the Creative Commons Attribution-NonCommercial-ShareAlike 3.0 License, which allows others to remix, tweak, and build upon the work non-commercially, as long as the author is credited and the new creations are licensed under the identical terms.

For reprints contact: reprints@medknow.com

How to cite this article: Issac TG, Chandra SR, Gupta N, Rukmani MR, Deepika S, Sathyaprabha TN. Autonomic dysfunction: A comparative study of patients with Alzheimer's and frontotemporal dementia - A pilot study. J Neurosci Rural Pract 2017;8:84-8. 
important structures which get affected early in these conditions. ${ }^{[3]}$ The neurotransmitters involved in these regions are acetylcholine, adrenaline, and noradrenaline which can be impaired quite early in patients with FTD and AD. ${ }^{[3,4]}$ The literature on early autonomic dysfunction seen in FTD and AD is scarce. ${ }^{[3-5]}$ Cardiac dysfunction, urinary dysfunction, and thermoregulatory dysfunction have been previously reported in FTD, but investigations into the integrity of the autonomic nervous system in this condition have been limited and need detailed exploration. ${ }^{[3-5]}$ Hence, we decided to study the autonomic nervous system function and its abnormalities prevailing in patients with early FTD and $\mathrm{AD}$. We have made an attempt to know if there is any differential involvement of autonomic system in these two groups which might help as an additional adjuvant in early diagnosis and the presence of autonomic dysfunction might give us insight into the role of neurotransmitters in these areas in the pathogenesis of these conditions and aid in probable novel, therapeutic options.

\section{Patients and Methods}

Right-handed patients $>18$ years fulfilling the International Behavioral Variant FTD Criteria for probable behavioral variant FTD (bvFTD) and AD patients with Hindi Mental Status Examination (HMSE) >23 years were recruited after thorough history taking and clinical examination and neuroimaging studies. ${ }^{[3]}$ Patients with other dementias and other comorbidities (Type II diabetes mellitus, hypertension, thyroid disorders) which might affect the autonomic nervous system and noncooperative patients were excluded from the study. Age- and gender-matched healthy controls were also recruited for the study. Informed consent was taken from the patients and caregivers (in case of necessity), as well as from the age-matched controls. Heart rate variability (HRV) and conventional cardiac autonomic function testing were performed in the recruited participants. The parameters were analyzed using Lab chart 7 V1.1 software, AD Instruments, Bella Vista, Australia and compared with control population using appropriate statistical methods using Statistical Package for social Sciences (SPSS) version 15.0 software, IBM Analytics, New York city ,USA.

\section{Results}

Out of the six patients with bvFTD, the female to male ratio was $1: 1$, with a mean age of $54.3 \pm 8.2$ years. Mean heart rate in our population was $77.33 \pm 6.5$ beats $/ \mathrm{min}$, mean systolic blood pressure (BP) was $132.16 \pm 13.8 \mathrm{~mm}$ of $\mathrm{Hg}$, and mean diastolic BP was $87.3 \pm 14.08 \mathrm{~mm}$ of $\mathrm{Hg}$, which was in the normal range. The mean HMSE score of the patients was $26 \pm 2.0$, with the mean duration of onset of illness being $3.1 \pm 2$ years. The magnetic resonance imaging in all the patients was suggestive of asymmetrical bifrontal and temporal lobe atrophy, with atrophy being significant on the right side. The patient to control ratio was 2:1. Mean overall total power was significantly low in the FTD group (564.86 $\mathrm{ms}^{2}$ ) as compared to the control group (2337.47 $\left.\mathrm{ms}^{2}\right)(P<0.001)$ [Table 1]. Significant reduction in the standard deviation of normal-to-normal intervals and root mean square of successive differences $(P<0.001)$ was also seen among the patient population. Significantly, altered sympathovagal balance showing sympathetic dominance was seen in the FTD group $(P=0.04)$ as compared to the age-matched controls [Figure 1a and b]. Definitive cardiac autonomic dysfunction was also observed in $66.7 \%$ of the patients tested. Valsalva maneuver and isometric hand grip test were abnormal in $67 \%$ of the patients, whereas orthostatic standing test was normal in all the patients in whom the test was done. Personality changes in the form of occasional anger outbursts, agitation, and apathy; disinhibited behavior was seen in all the patients. Majority of these patients $(67 \%)$ had features of food faddism with preference for carbohydrate-rich food items, and of them, 33.3\% had change in food preference with more consumption of nonvegetarian food items indicating ventral insular and hypothalamic pathology.

With reference to $A D$, there were 25 patients and 25 controls. Their gender distribution was 14 males and 11 females [Figure 2]. Mean age was $62.28 \pm 6.87$ and mean clinical dementia rating score was 9.82 . Imaging showed medial temporal and biparietal atrophy. Recent memory impairment was present in all the patients, visuospatial disorientation present in $76 \%$, language impairment in $60 \%$, and executive dysfunction and impaired social and occupational function in $100 \%$. The common symptoms were apraxias (48\%), agnosias (16\%), and psychiatric

Table 1: Overall total power being significantly low in the frontotemporal dementia group

\begin{tabular}{lccc}
\hline${\text { (in } \text { s }^{2} \text { ) }}^{\text {LF }}$ & HF & Total Power \\
\hline Patient 1 & 213.7 & 56.8 & 659 \\
Patient 2 & 207.81 & 111.87 & 556.64 \\
Patient 3 & 87.89 & 118.7 & 345.66 \\
Patient 4 & 241 & 52 & 1006 \\
Patient 5 & 254 & 200 & 669 \\
Patient 6 & 35.4 & 5.8 & 152 \\
Control 1 & 586.69 & 1312.22 & 5105 \\
Control 2 & 210.7 & 199.3 & 702 \\
Control 3 & 235.54 & 317.94 & 317.94 \\
\hline
\end{tabular}



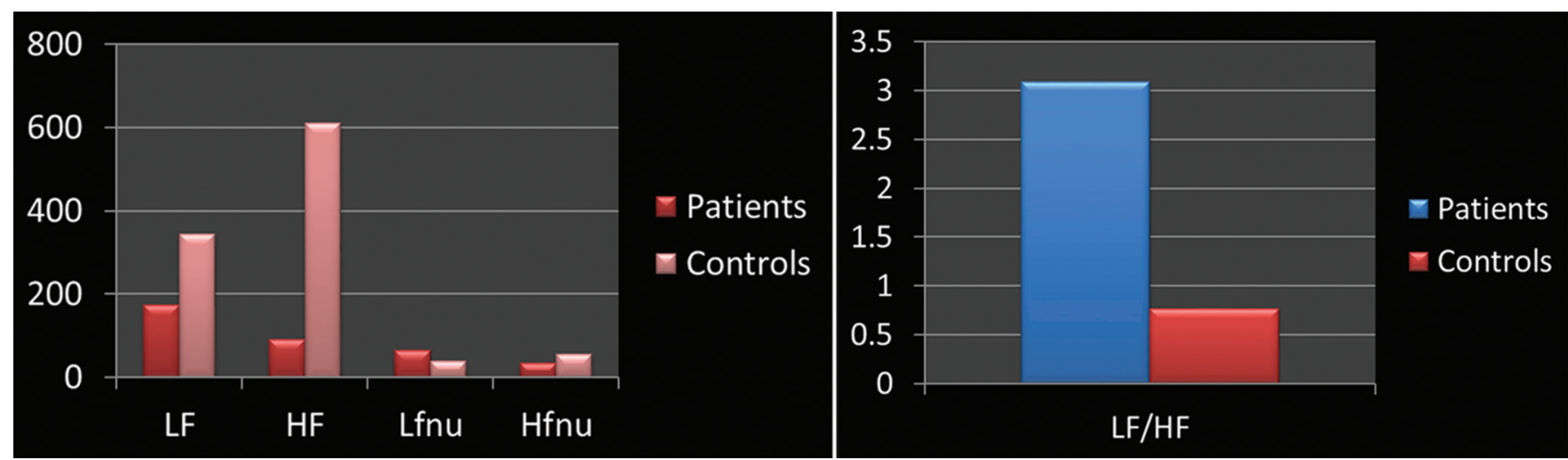

Figure 1: (a) Elevated high frequency values as compared to low frequency values suggestive of poor parasympathetic balance. (b) High low frequency/high frequency ratio indicating sympathetic predominance in patients when compared with age-matched controls

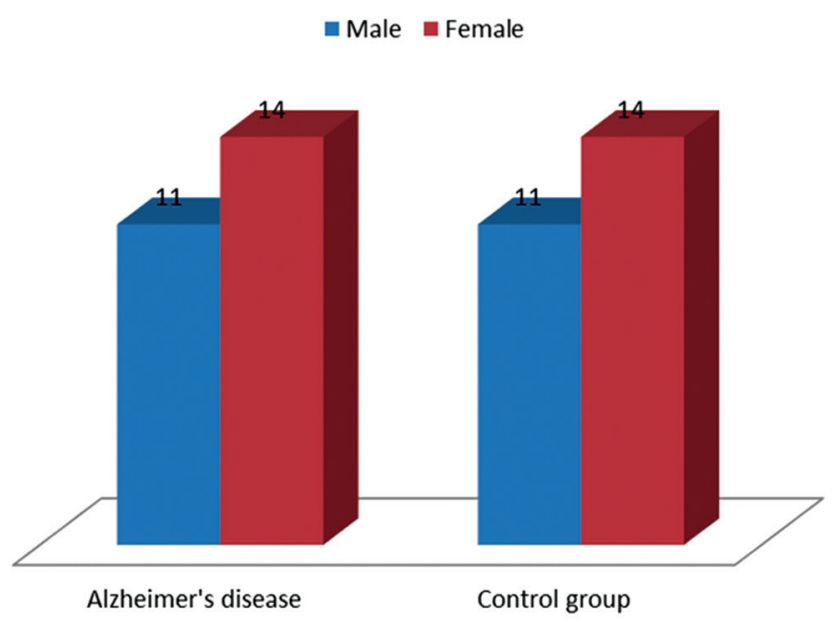

Figure 2: Alzheimer's disease - graph showing gender difference

symptoms (32\%), respectively. With reference to patients with $\mathrm{AD}$, out of various time and frequency domain parameters, low frequency (LF) $(P=0.017)$ and high frequency $(\mathrm{HF})(P=0.012)$ normalized units (n.u.) and LF/HF ratio $(P=0.014)$ showed statistical significance. High LF n.u. and LF/HF ratio were suggestive of sympathetic predominance. There is statistically significant frequency domain.

Compared to controls group, Alzheimer disease group has low HF n.u. value which denotes parasympathetic suppression and domain parameters found in HRV analysis are suggestive of sympathetic predominance and suppression of parasympathetic activity in Alzheimer's group [Tables 2 and 3]. Therefore, early evaluation of autonomic functions is helpful to understand the extent of the structures involved other than the cognitive network. The pattern of involvement is similar in both FTD and AD. Therefore, akin to temporal lobe involvement which is common to both diseases autonomic involvement is also a shared territory. This information might pave the way for further research into the etiopathological similarities and differences in these two disorders which may contribute to novel therapeutic options in future. Parasympathetic suppression seen in AD patients as against FTD can be explained by cholinergic deficiency in this condition. ${ }^{[6,7]}$

\section{Discussion}

Patients with early FTD and AD are often misdiagnosed as psychiatric illness, "mid-life crisis," and even only as Alzheimer's disease. Thus, biomarkers for early detection and prognostication in cortical dementias are of profound clinical implications. ${ }^{[3,5,8,9]}$ In our study, patients with bvFTD were recruited because they constitute the most common clinical variant of FTD and have a better cognition and devoid of language disabilities unlike other variants making them better candidates. Our patients with bvFTD, though independent for activities of daily living, showed abnormality across most of the domains of autonomic functions when compared to control subjects even though they were clinically asymptomatic for autonomic dysfunction. Unlike previous studies, our study showed increased sympathovagal balance with sympathetic predominance in both conditions. Bradycardia was not seen as a prominent feature. ${ }^{[6]}$ Reduced power was also a consistent finding in our study which indicates an early involvement of central autonomic centers in these patients as compared to control population. This study reveals that autonomic dysfunction can be an important manifestation in patients with early FTD and AD. It also shows that there is significant increase in the sympathovagal balance, indicating sympathetic dominance. The presence of definitive cardiac autonomic dysfunction in these groups of patients also emphasizes the importance of autonomic centers such as insula and hypothalamus which can get affected in these neurodegenerative diseases. It is well 
Table 2: Autonomic function test parameters in controls versus Alzheimer's dementia group

\begin{tabular}{|c|c|c|c|c|c|c|c|c|c|c|}
\hline & \multicolumn{5}{|c|}{ Control group } & \multicolumn{5}{|c|}{ Alzheimer's group } \\
\hline & $n$ & Median & Range & Minimum & Maximum & $n$ & Median & Range & Minimum & Maximum \\
\hline RMSSD & 25 & 18.7900 & 73.86 & 6.38 & 80.24 & 25 & 17.5500 & 72.36 & 4.05 & 76.41 \\
\hline SDNN (ms) & 25 & 30.2400 & 65.93 & 11.84 & 77.77 & 25 & 34.1800 & 64.39 & 12.01 & 76.40 \\
\hline NN50 & 25 & 2.00 & 134 & 0 & 134 & 25 & 4.00 & 102 & 0 & 102 \\
\hline pNN50 & 25 & 41.00 & 48.55 & 0.00 & 48.55 & 25 & 1.1300 & 28.80 & 0.00 & 28.80 \\
\hline Total power $\left(\mathrm{ms}^{2}\right)$ & 25 & 825.7700 & 9091.84 & 138.16 & 9230.00 & 25 & 1011.1300 & 5605.23 & 125.77 & 5731.00 \\
\hline LF power & 25 & 182.5100 & 3037.40 & 17.60 & 3055.00 & 25 & 286.2800 & 1565.76 & 24.24 & 1590.00 \\
\hline HF power & 25 & 109.0100 & 3359.14 & 9.86 & 3369.00 & 25 & 81.3900 & 932.88 & 5.12 & 938.00 \\
\hline LF (n.u.) & 25 & 44.55 & 65.49 & 18.42 & 83.91 & 25 & 65.40 & 77.95 & 18.81 & 96.76 \\
\hline HF (n.u.) & 25 & 42.86 & 56.33 & 14.88 & 71.21 & 25 & 26.44 & 59.39 & 2.89 & 62.28 \\
\hline LF/HF ratio & 25 & 1.05000 & 5.260 & 0.370 & 5.630 & 25 & 2.45000 & 33.128 & 0.302 & 33.430 \\
\hline
\end{tabular}

Table 3: Heart rate variability parameters in Alzheimer's dementia

\begin{tabular}{lcc}
\hline Variables & $\begin{array}{c}\text { Mann-Whitney } \\
\text { U-test }\end{array}$ & $\begin{array}{c}\text { Asymptotic } \\
\text { significant (two-tailed) }\end{array}$ \\
\hline SDNN (ms) & 283.0 & 0.567 \\
RMSSD & 295.0 & 0.734 \\
NN50 & 308.0 & 0.929 \\
pNN50 & 308.0 & 0.929 \\
Total power $\left(\mathrm{ms}^{2}\right)$ & 277.0 & 0.491 \\
LF power & 262.0 & 0.327 \\
HF power & 263.0 & 0.337 \\
LF (n.u.) & 189 & 0.017 \\
HF (n.u.) & 183 & 0.012 \\
LF/HF ratio & 185.5 & 0.014
\end{tabular}

HF: High frequency, LF: Low frequency, RMSSD: Root mean square successive difference, SDNN: Standard deviation of normal-to-normal, n.u.: normalized units

known that cholinergic deficiency (parasympathetic) seen in patients with Alzheimer's disease but not in patients with FTD but information on whether there is any sympathetic: Parasympathetic imbalance is scarce in literature. Interventions aimed at reducing sympathetic hyperactivity and increasing parasympathetic activity could be beneficial in control of behavioral symptoms and need to be evaluated. The role of selective serotonin reuptake inhibitors, small doses of beta blockers, and nonsedative anxiolytics such as buspirone may be of great potential in some of these cases which require further validation. ${ }^{[8-10]}$ Early interventions which can improve parasympathetic integrity such as yoga, meditation, and relaxation exercises can prove to be important nonpharmacologic treatment modalities in FTD and AD. Based on our results, techniques which increase the parasympathetic drive such as noninvasive vagal nerve stimulation and specific exercises which can augment vagal tone can be of potential utility and need further exploration. In patients with $\mathrm{AD}$, there is sympathetic dominance, but there is also parasympathetic suppression which correlates with the neurochemical differences in these two conditions and could serve as a diagnostic tool. Involvement of ANS in both conditions might help us to research into shared factors in the degenerative process which might give etiological and therapeutic clues in future. Treatment modalities such as yoga which improve parasympathetic function and suppress sympathetic will become a valuable tool in both varieties of cortical dementias in the early stage.

\section{Conclusion}

This pilot study reveals that autonomic dysfunction can be an important manifestation in patients with early FTD and AD. It also shows that there is a significant change in the sympathovagal balance in the form of sympathetic dominance in both groups and parasympathetic suppression in AD. This differential suppression of parasympathetic can be an early diagnostic tool in differentiating both. The sympathetic dominance seen in both groups might indicate interesting shared etiological factors which might point to novel causal and thus therapeutic options such as yoga which improves parasympathetic functions and suppresses sympathetic dominance. However, the number is too small in view of strict exclusion of mixed dementias and therefore needs to be evaluated in a larger population.

\section{Financial support and sponsorship Nil.}

\section{Conflicts of interest}

There are no conflicts of interest.

\section{References}

1. Mercy L, Hodges JR, Dawson K, Barker RA, Brayne C. Incidence of early-onset dementias in Cambridgeshire, United Kingdom. Neurology 2008;71:1496-9.

2. Alladi S, Mekala S, Chadalawada SK, Jala S, Mridula R, Kaul S. Subtypes 
of dementia: A study from a memory clinic in India. Dement Geriatr Cogn Disord 2011;32:32-8.

3. Ahmed RM, Iodice V, Daveson N, Kiernan MC, Piguet O, Hodges JR. Autonomic dysregulation in frontotemporal dementia. J Neurol Neurosurg Psychiatry 2015;86:1048-9.

4. Meel-van den Abeelen AS, Lagro J, Gommer ED, Reulen JP, Claassen JA. Baroreflex function is reduced in Alzheimer's disease: A candidate biomarker? Neurobiol Aging 2013;34:1170-6.

5. den Abeelen AS, Lagro J, van Beek AH, Claassen JA. Impaired cerebral autoregulation and vasomotor reactivity in sporadic Alzheimer's disease. Curr Alzheimer Res 2014;11:11-7.

6. Vermeij A, Meel-van den Abeelen AS, Kessels RP, van Beek AH, Claassen JA. Very-low-frequency oscillations of cerebral hemodynamics and blood pressure are affected by aging and cognitive load. Neuroimage 2014;85(Pt 1):608-15.

7. Issac TG, Chandra SR, Nagaraju BC. Transcranial magnetic stimulation in patients with early cortical dementia: A pilot study. Ann Indian Acad Neurol 2013;16:619-22.

8. Conner R, Sheikh M, Grubb B. Postural orthostatic tachycardia syndrome (POTS): Evaluation and management. Br J Med Pract 2012;5:a540.

9. Kamal S, Sturman S, Sarkar PK, Michael A. Postural orthostatic tachycardia syndrome (POTS): A diagnostic dilemma. Br J Cardiol 2010;17:36.

10. Beach PA, Huck JT, Miranda MM, Bozoki AC. Autonomic, behavioral, and subjective pain responses in Alzheimer's disease. Pain Med 2015;16:1930-42. 